\title{
Influence of the thermomechanical properties on the joining of adhesion incompatible polymers by form-fit using the vibration welding process
}

\author{
M. Wolf", S. Hertle, D. Drummer \\ Friedrich-Alexander-Universität Erlangen-Nürnberg (FAU), Faculty of Engineering, Department of Mechanical \\ Engineering, Institute of Polymer Technology, Am Weichselgarten 9, 91058 Erlangen, Germany
}

Received 21 September 2018; accepted in revised form 26 November 2018

\begin{abstract}
In the product manufacturing cycle, the joining of polymers often takes a central position. Vibration welding is characterized by short cycle times, high process flexibility and stability as well as by the achievement of high bond strengths without using additives. However, the vibration welding process is clearly restricted by a necessary adhesion compatibility of the joining partners. By means of structuring one joining partner and subsequent filling of the structures by a second joining partner, the joining by form-fit using vibration welding enables the combination of adhesion incompatible polymers. Within these investigations, bonds between adhesion incompatible semi-crystalline thermoplastics were generated based on mechanical adhesion. Also semi-crystalline and amorphous polymers could be connected for the first time without any connecting elements, although they conventionally do not show mechanical resilience. The analysis of the thermomechanical material properties and the resulting multimaterial link enabled the determination of first influences on the resulting formfit connection caused by the occurring joining zone temperature.
\end{abstract}

Keywords: processing technologies, vibration welding, joining by form-fit, mechanical adhesion, adhesion incompatible

\section{Introduction}

The joining of polymers often occupies a central position within the manufacturing process chain. By connecting several components, it is possible to circumvent existing restriction of the primary shaping process (e.g. in injection molding or extrusion) [1]. The common industrially applied joining methods for thermoplastics are welding, adhesive bonding and mechanical fastening [2,3]. Welding is characterized especially by the possibility of producing parts with high joint strength without the use of additives [4]. Thus, welding processes provide great advantages compared to adhesive bonding or mechanical connections. The vibration welding process in particular is characterized by short cycle times, high reproducibility and process stability as well as the suitability for large joining surfaces [5].

Nevertheless, the process is limited to joining of adhesion compatible material combinations. This leads to a restriction on material selection in the design process or the use of expensive designs or processes. Consequently, part functionalization as well as the creation of locally adapted product properties are strongly narrowed. In order to realize joints between adhesion incompatible materials, adhesive bonds are often used. Admittedly, these bonds are severely hindered due to long cycle times and thus assembly rate limitations, restricted introduction of force (avoidance of peel and bend loads), and commonly harmful ingredients $[6,7]$. Therefore, the aim of the paper is 
the extension of form-fit connections by welding to join adhesion incompatible polymer combinations of semi-crystalline and amorphous thermoplastics. Furthermore, the influence of the thermomechanical properties of the used materials is investigated on the form-fit based connection. These can affect the dimensional stability of the structures as well as the filling behavior of the undercuts in the joining process. Within these investigations, a circumvention of the existing material restrictions in multimaterial combinations as well as first conclusions on existing interactions between material, form-fit welding process and resulting part characteristics can be drawn.

\subsection{Vibration welding}

The linear vibration welding process is based on an oscillating friction-relative movement of the joining partners [4]. Due to the compression of the components during the movement with the amplitude a and the applied friction force $F_{\mathrm{R}}$, melting (semi-crystalline) or softening (amorphous) of the polymers takes place and a welding pressure-induced melt flow occurs in the joining zone [4]. At the end of the friction-relative movement, the joining partners cool down under pressure and a connection is formed [8].

Fundamental investigations on the vibration welding of thermoplastics have shown a characteristic course of the resulting weld path, regardless of the part geometry, material and process parameters [4, 9-11]. This course can be divided into the four typical phases of solid friction (phase 1), transient melt formation (phase 2), steady-state melt flow (phase 3), and cooling (phase 4). The achievement of the third phase is considered to be a quality criterion of vibration weld joints [12] and is required to achieve high weld strengths $[10,13,14]$. Besides, during the steady-state phase a constant temperature in the joining zone occurs due to the existing energy equilibrium [11]. These self-regulating process properties were confirmed in experimental studies $[15,16]$. Vetter and Ehrenstein [15] was able to demonstrate a maximum joining zone temperature of $20 \mathrm{~K}$ above the melting point for polypropylene. For vibration welding of polyamide 66, Bates et al. [16] detected a temperature of 5-15 K above the onset of the melting temperature. In addition to the joining of similar materials, bonds consisting of different polymer types are industrially of great interest. These multimaterial connections are particularly challenging due to the process- and material-related limitations in welding processes. Beside a similar melting temperature and viscosity of the materials, a compatibility of the thermoplastics to each other is necessary in order to generate a link $[8,17]$. This required adhesion compatibility severely restricts the possible polymer combinations and inhibits the industrial spread of multimaterial connections produced by welding. So-called compatibility matrices are provided for joining technology e.g. by Rotheiser [6]. These matrices show that connections between semi-crystalline and amorphous thermoplastics are feasible only in exceptional cases and furthermore, the production of joints between standard thermoplastics and technical thermoplastics, e.g. between polyolefins and polyamides are very limited.

\subsection{Joining of incompatible material combinations \\ Conventional joining methods}

Polymer combinations, which have no adhesion compatibility, show very limited bonding options. For adhesion incompatible polymer-polymer combinations, a cohesive joint can only be generated by adhesive bonding technologies. The resulting connection is based on chemical bonds and intermolecular forces as van der Waals forces, hydrogen bonds, dipole connections, induction or dispersion forces [18], a mechanical interlocking between joining partner and adhesive [1] and, where appropriate, a mutual penetration of polymer molecules and the adhesive [1]. Adverse effects during adhesive bonds include, inter alia, the required time-consuming and expensive surface pretreatment of the joining partners (e.g. cleaning, roughening, activation) $[1,18]$ as well as healthendangering ingredients. In addition, a customized adhesive often needs to be developed and approved. Form-fit connections represent an alternative to combine adhesion incompatible polymers. In this case, an interlocking or meshing of the joining partners takes place, which enables a force transmission without material interactions across the joining interface [1]. Methods for generating joints by form-fit are e.g. overmolding in assembly injection molding, rivet joints or snap fits $[1,19]$. Disadvantages of these connections are the complex and expensive tools in the assembly injection molding, the design of the joining zone (breakthroughs or overlap of the joining partners) and the limited freedom of geometry, which restrains an industrial use of these methods. 
Two-stage joining processes with structuring one joining partner

Due to the strong limitation of possible material combinations in welding processes and existing restrictions for alternative joining technologies (adhesive bonding, riveting, assembly injection molding etc.), it is necessary to develop new process technologies that allow the joining of adhesion incompatible polymers. Therefore, investigations of dissimilar material combinations, especially in the area of polymermetal combinations [20-24], have shown a high potential for joints based on or provided by form-fits in a two-stage process with pre-structuring the metal part. Furthermore, initial investigations [25] into formfit polymer-polymer combinations demonstrated the feasibility and potential of mechanical adhesion in welding for expanding the material combination spectrum. Hereby, functional lap joints, which can transmit and endure measurable loads, could be produced for the adhesion incompatible combinations of polypropylene with polyamide. The form-fit joining by welding can be divided into the structuring process and the joining process, Figure 1. Both process steps are conducted with the help of the vibration welding technology. The use of the occurring oscillating movement in X-axis enables the generation of undercuts additionally to the conventional welding process. During the structuring process, a friction relative movement takes place between a structuring tool, which contains pins with undercuts, and the structured-partner. Due to the introduced friction energy, melting of the polymer occurs. The applied structuring pressure displaces the molten material into the structures of the tool. Upon reaching a selected structuring depth or time, the oscillating movement stops and the tool is removed in $z$-direction from the still molten plastic. After the structuring process, the structuring tool is replaced by the bonding-partner and a friction-relative movement takes place again. The bonding-partner melts due to the introduced friction energy and a filling of the generated pins in the structured-partner takes place. Therefore, the material of the structuredpartner should not soften and keep its dimensional

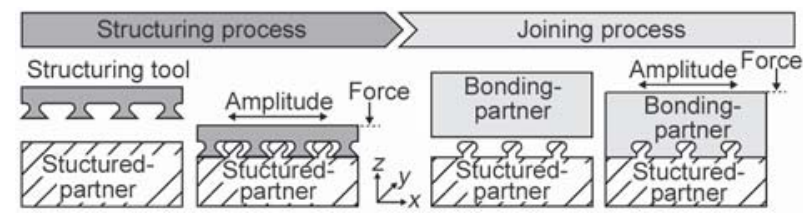

Figure 1. Process schema of joining by form-fit using the vibration welding technology [25]. stability during the joining process. Otherwise, a destruction of the existing pins and undercuts may arise. After a defined joining depth or time, the friction movement stops, the molten polymer solidifies under pressure and a form-fit connection is generated [25]. Initial experiments have been able to demonstrate the high potential of form-fit connections between adhesion incompatible semi-crystalline thermoplastics produced in welding. However, the resulting interactions between pin geometry, process parameters and bond quality as well as the material influences (e.g. thermal properties, viscosity, stiffness) on the process and the resulting joints were not investigated. With the help of this study, on the one hand the joining by form-fit in the vibration welding process is extended to adhesion incompatible connections between semicrystalline and amorphous thermoplastics for the first time. On the other hand, novel investigations of the material influence on the form-fit joining process are conducted. Particular attention is paid to changes of the previously introduced pin-geometry caused by the joining process with varied materials featuring different thermomechanical properties.

\section{Experimental section}

\subsection{Materials and test specimens \\ Used materials}

For the conducted research, a commercial available polyamide 66 (PA66), type Durethan A30S (Lanxess Deutschland $\mathrm{GmbH}$, Cologne, Germany) was used as structured-partner. To investigate the influence of the thermomechanical material properties on the form-fit joining process, the commercial semi-crystalline polypropylene (PP), type Sabic 505P (Sabic Europe B. V., Sittard, Netherlands) as well as the commercial amorphous polymethylmethacrylate (PMMA), type Plexiglas 7N (Evonik Industries AG, Essen, Germany) and polycarbonate (PC), type Makrolon 2805 (Covestro AG, Leverkusen, Germany) were used as bonding-partner.

First, plates of all materials with geometrical dimension of $115 \mathrm{~mm} \times 115 \mathrm{~mm} \times 4 \mathrm{~mm}$ were produced by injection molding using a machine type Allrounder 370V/800-315 (Arburg GmbH \& Co. KG, Loßburg, Germany) with the process parameters listed in Table 1. The PA66 granulate was dried at $80^{\circ} \mathrm{C}$ for $6 \mathrm{~h}$ before the injection molding process. Subsequently, test specimens for the welding trials with geometrical dimensions of $50 \mathrm{~mm} \times 50 \mathrm{~mm} \times 4 \mathrm{~mm}$ were milled out of the plates. These specimens were used for 
Table 1. Processing parameters of injection molding process.

\begin{tabular}{|l|c|c|c|c|c|}
\hline Material & $\begin{array}{c}\text { Temperature profile } \\
\mathbf{s c r e w ~} \rightarrow \text { nozzle } \\
{\left[{ }^{\circ} \mathbf{C}\right]}\end{array}$ & $\begin{array}{c}\text { Mold temperature } \\
\text { ejector/nozzle } \\
{\left[{ }^{\circ} \mathbf{C}\right]}\end{array}$ & $\begin{array}{c}\text { Injection speed } \\
{[\mathbf{m m} / \mathbf{s}]}\end{array}$ & $\begin{array}{c}\text { Holding pressure } \\
{[\mathbf{b a r}]}\end{array}$ & $\begin{array}{c}\text { Holding pressure } \\
\text { time } \\
{[\mathbf{s}]}\end{array}$ \\
\hline PA66 & $280 / 280 / 270 / 260 / 250$ & $85 / 80$ & 50 & 500 & 30 \\
\hline PP & $250 / 240 / 230 / 220 / 210$ & $60 / 60$ & 60 & 250 & 40 \\
\hline PMMA & $230 / 220 / 210 / 200 / 190$ & $60 / 65$ & 60 & 450 & 25 \\
\hline PC & $300 / 290 / 280 / 270 / 260$ & $95 / 95$ & 60 & 500 & 30 \\
\hline
\end{tabular}

joining the adhesion incompatible polymers by butt joints.

\section{Structuring tool}

To enable the generation of pin structures with undercuts in the structured-partner, a structuring tool with 16 pins on one side and a thickness $t$ of $2 \mathrm{~mm}$ was used. The pins were machined into the tool steel by laser cutting. The relevant dimensions of the used tool are given in Figure 2. Contrary to conventional butt joints with a joining area of $200 \mathrm{~mm}^{2}$ (Equation (1)), the structured-partner has an increased theoretical joining surface of approx. $308 \mathrm{~mm}^{2}$ ( Equation (2)-(6)). However, only the undercut areas, which are perpendicular to the joining zone, are effective for the form-fit connection. So an area of approx. $22 \mathrm{~mm}^{2}$ (Equation (7)) is available for transmission of forces in $z$-direction:

$$
\begin{aligned}
A_{\text {conventional }}= & 50 \mathrm{~mm} \cdot 4 \mathrm{~mm} \\
A_{\text {structured partner }} & =(16 u+16 b) t+ \\
& +50 \mathrm{~mm} \cdot(4 \mathrm{~mm}-t) \\
\text { with: } u= & r\left[(180+2 \alpha) \cdot \frac{\pi}{180}\right] \\
\text { and: } \alpha= & \arcsin \left(\frac{0.75 \mathrm{~mm}}{r}\right) \\
\text { and: } b= & 3 \mathrm{~mm}-2 r+2(r-a) \\
\text { and: } a= & \sqrt{r^{2}-(0.75 \mathrm{~mm})^{2}}
\end{aligned}
$$

$$
A_{\text {undercut }}=32(r-a) t
$$

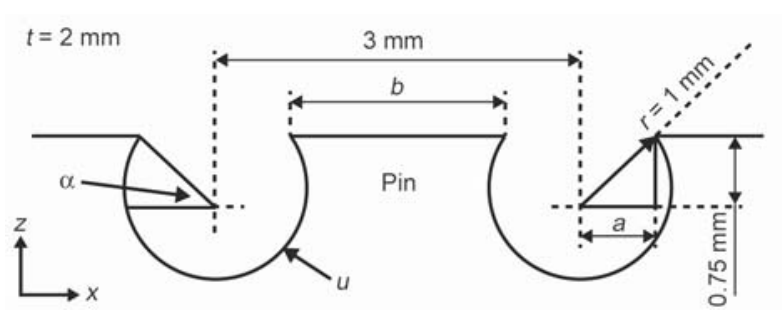

Figure 2. Relevant dimensions of the structuring tool for form-fit butt joints.

\subsection{Test methods}

\section{Vibration welding process}

For the production of the form-fit connections, the structuring process and the subsequent joining process were performed on a linear vibration welding machine Branson M-112 HR (Branson Ultraschall, Dietzenbach, Germany). Furthermore, conventional welding tests were carried out on the same machine without structuring a joining partner. To determine the temperature in the weld area during conventional welding tests, thermocouples of type $\mathrm{K}$ were used. The thermocouples with a diameter of $0.2 \mathrm{~mm}$ were positioned in the center of the joining zone directly between the welding plates. The temperature measurements were carried out with a repetition number of three. For investigations with polypropylene Vetter and Ehrenstein [15] showed an influence of this joining zone temperature mainly by the viscosity of the material and the amplitude during the friction movement. Bates et al. [16] determined for polyamide 66 joints an influence of the weld temperature by the joining pressure. Although an influence on the resulting joining surface cannot be excluded, a comparability of the observed temperatures can be assumed.

Table 2. Parameter settings for vibration welding process (repetition number $n=5$ ).

\begin{tabular}{|l|l|c|c|c|c|}
\hline \multicolumn{2}{|c|}{ Process } & $\begin{array}{c}\text { Frequency } \\
{[\mathbf{H z}]}\end{array}$ & $\begin{array}{c}\text { Amplitude } \\
{[\mathbf{m m}]}\end{array}$ & $\begin{array}{c}\text { Force } \\
{[\mathbf{N}]}\end{array}$ & $\begin{array}{c}\text { Joining path } \\
{[\mathbf{m m}]}\end{array}$ \\
\hline \multirow{2}{*}{ Joining by form-fit } & Structuring & 235 & 0.9 & 350 & 1.0 \\
\cline { 2 - 6 } & Joining & 235 & 0.9 & 400 & 1.3 \\
\hline Conventional vibration welding & 235 & 0.9 & 400 & 1.3 \\
\hline
\end{tabular}


All parameter settings used for the tests are listed in Table 2. The frequency is determined by the resonance frequency of the welding system. The amplitude and fore was set in preliminary tests so that the tests could be performed entirely without influences. The joining path was chosen, to fully form the pin structures and subsequently a sufficient joining path is completed in order to fill the generated hollows by the bonding-partner.

\section{Light microscopic investigations}

Microscopic investigations were carried out to investigate the generated pin structures and possible undercuts of the structured-partner after the structuring process. Furthermore, the resulting structure filling and produced multimaterial combination after the second process step was examined. The samples were analyzed by reflected light microscopy with a magnification of 2.5. In order to avoid thermal influences while preparing the specimens, the weld areas were cut out of the welded plates with a watercooled low-revolution saw and subsequently embedded in cold-curing epoxy resin.

\section{Differential scanning calorimetry (DSC)}

For DSC measurements with a device of type DSC Q 2000 (TA Instruments, New Castle, US), specimen quantities of approx. 3-5 mg were prepared from the cross-section of the welding plates. The applied heating and cooling rates were $10 \mathrm{~K} / \mathrm{min}$ in a nitrogen atmosphere. In order to analyze the melting (semicrystalline) or softening (amorphous) behavior, the behavior of the specimens was evaluated during initial heating.

\section{Fast scanning calorimetry (FSC)}

FSC analyses were performed using a Flash DSC 1 (Mettler-Toledo GmbH, Columbus, US). Test samples were prepared from a $10 \mu \mathrm{m}$ thin cut (of the specimens joining zone) using a scalpel and placed on the measurement sensor of the FSC. The sample weight was too small to measure. Considering the dimension and density of the used polymers the weight could be estimated at about $50 \mathrm{ng}$. All measurements were conducted in a nitrogen atmosphere and the resulting heat flow rate was illustrated with respect to the temperature.

Due to the high heating rates in the vibration welding process, Bates showed heating rates of about $100 \mathrm{~K} / \mathrm{s}$ in his real time temperature measurements [16], the attainable heating rates in standard DSC are not sufficient to evaluate the process-relevant heating behavior in vibration welding. Therefore, different heating velocities of 100 and $500 \mathrm{~K} / \mathrm{s}$ were analyzed by FSC. For the different measurements, the sample was first heated up to $290^{\circ} \mathrm{C}$ (PP), $280^{\circ} \mathrm{C}$ (PMMA) and $300^{\circ} \mathrm{C}$ (PC) and then cooled down to $-20^{\circ} \mathrm{C}$ with a cooling rate of $1 \mathrm{~K} / \mathrm{s}$ to achieve a similar state as in injection molded parts $[26,27]$. The prior heating is necessary so that the very small sample quantity contacts with the sensor adequately. Subsequently, cooling must take place below the glass transition. In order to have comparable initial conditions, all samples were cooled down to the same temperature. Afterwards, the sample was heated up in a second heating step to get a comparable heating rate as in the welding process.

\section{Rotational rheometry}

The rheological behavior of the material was characterized using a rotational rheometer of type AR 2000 (TA Instruments, New Castle, US). The measurements were carried out in oscillating plate-toplate measurements on specimen with diameter of $25 \mathrm{~mm}$ at frequencies of 1,25 and $50 \mathrm{~Hz}$ and strain of $0.1 \%$. The investigated temperatures were 130 to $210^{\circ} \mathrm{C}$ (PP) and 200 to $260^{\circ} \mathrm{C}$ (PMMA, PC) in temperature steps of $10 \mathrm{~K}$. The frequency and temperature dependent viscosity $\eta$ as well as the storage modulus $G^{\prime}$ and loss modulus $G^{\prime \prime}$ of the semi-crystalline and amorphous materials were analyzed. The investigation of the entropy-elastic material behavior as well as the temperature-dependent viscosity can allow an estimation of the resulting joining temperature and thus about the occurring thermal influence on the generated pin structures. Furthermore, an explanation of the interactions between the filling behavior of the structures and the thermomechanical properties of the bonding-partner can be accomplished.

\section{Mechanical testing (tensile test)}

Tensile tests were carried out to determine the mechanical load capability of the multimaterial combinations. For this purpose, a universal testing machine with computer-controlled test executions, type Zwick 1484 (Zwick GmbH \& Co. KG, Ulm, Germany) was used and the maximum achievable tensile strength was evaluated. The mechanical tests were performed under standard conditions according to DIN EN ISO $219\left(T=23^{\circ} \mathrm{C}\right.$, relative air humidity: $\left.50 \%\right)$ with a 
pull-off speed of $1 \mathrm{~mm} / \mathrm{min}$ and a repetition number of five. Before testing, the specimens were stored dry in a vacuum oven at $23^{\circ} \mathrm{C}$ to avoid any moisture absorption.

In contrast to previous investigations [25] on lap joints of PA66-HDPE, the butt joints have a uniaxial tensile load without any torsion that acts directly in pin direction. Due to this uniaxial load and the avoidance of any torsion, an assessment of the transmitted forces due to the form-fit can be conducted.

\section{Fracture behavior}

The fracture surfaces were investigated after the mechanical testing to evaluate the fracture behavior of the multimaterial combinations depending on the used material (ductility, strength, young's modulus) of the bonding-partner. For this reason, a stereomicroscope type Discovery.V12 (Carl Zeiss AG, Oberkochen, Germany) was used to analyze the PA66 surface of the conventional weld joints. Additionally, the fracture behavior of the form-fit connections was examined using a scanning electron microscope (SEM) of type Gemini Ultra-Plus (Carl Zeiss AG, Oberkochen, Germany).

\section{Results and discussion}

\subsection{Conventional vibration welding process}

\section{Temperatures in the weld zone}

The evaluation of the temperature measurements during the conventional vibration welding process is shown in Figure 3a. The curves show the typical temperature profile with a constant joining zone temperature during the third process phase. Hereby, the material of the bonding-partner and its thermomechanical properties mainly influence the resulting constant temperature. As seen later, the melting peak temperature of the used PP is $162^{\circ} \mathrm{C}$, whereas the melting peak temperature of the used PA66 is $261{ }^{\circ} \mathrm{C}$. The glass transition for the amorphous thermoplastics are approximately at $108^{\circ} \mathrm{C}$ for PMMA and $146^{\circ} \mathrm{C}$ for PC. The measurements in the weld zone show that a constant temperature in the joining zone of $174 \pm 1^{\circ} \mathrm{C}$ appear for multimaterial connections between PA66 and PP. This temperature is consistent with investigations by Vetter and Ehrenstein [15], who detected a temperature of maximum $20 \mathrm{~K}$ above the melting temperature for PP. For connections between PA66 and PMMA a constant temperature in the joining zone of $228 \pm 4{ }^{\circ} \mathrm{C}$ and for PA66-PC joints of $242 \pm 2{ }^{\circ} \mathrm{C}$ occurs during the welding trials.

The achieved heating rates, Figure $3 b$, at the beginning of the vibration welding process are for PA66$\mathrm{PP}$ joints in the range of $120-140 \mathrm{~K} / \mathrm{s}$, for PA66PMMA in the range of $260-300 \mathrm{~K} / \mathrm{s}$ and for PA66-PC at about $340-380 \mathrm{~K} / \mathrm{s}$.

\subsection{Form-fit joining by vibration welding process}

Generated pin structures

Figure 4 illustrates the pin structures of the polyamide 66, which are generated in the first process step of the form-fit joining process. The introduced structures have a height between $1.8-2.1 \mathrm{~mm}$ and a width of 1.5-1.8 mm. Compared to the theoretical pin structure with a height of $1.75 \mathrm{~mm}$ and a width of $2.0 \mathrm{~mm}$, the produced pins show a slight extension in $z$-direction. This can be due to the pull-out movement of the structuring tool at the end of the structuring process.
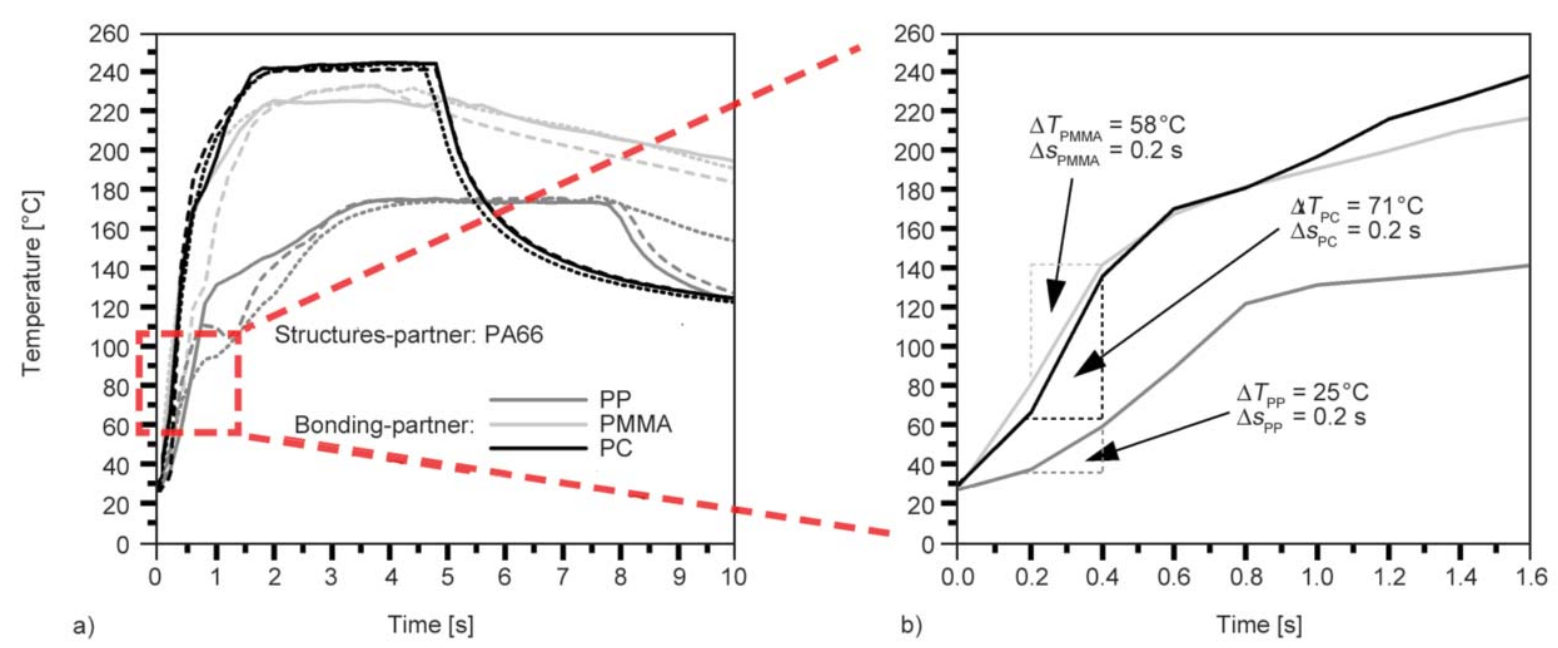

Figure 3. Temperature in weld zone of conventional vibration welding (a) and exemplary estimation of the resulting heating rate (b). 


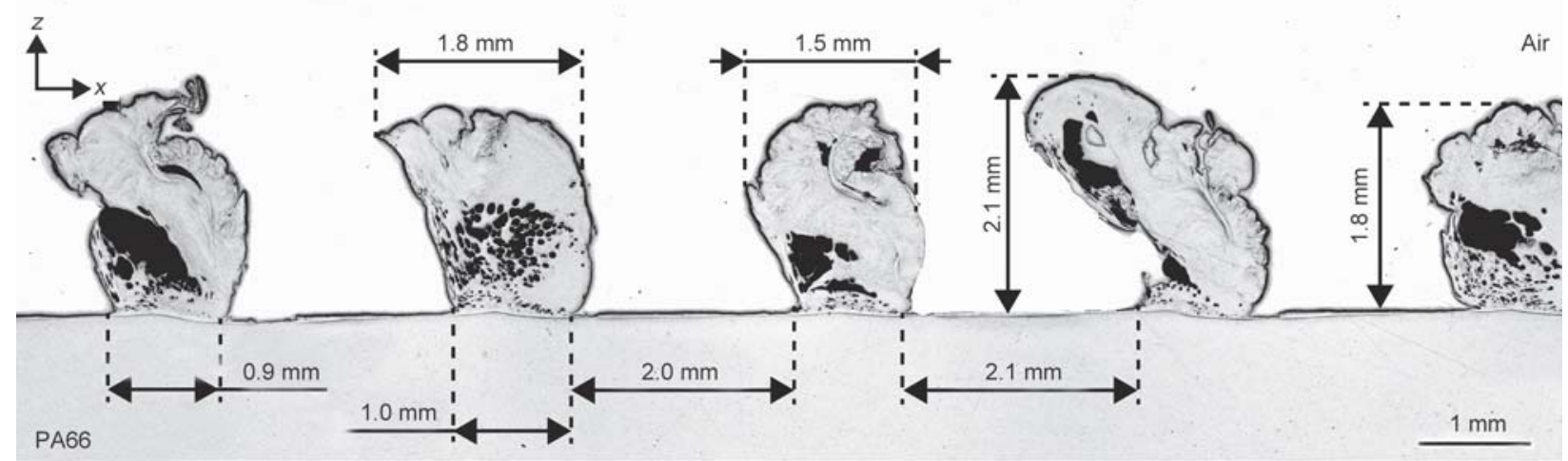

Figure 4. Microscopic image of generated pin structures in structured-partner (PA66).

In addition, the created pin structures exhibit undercuts in $z$-direction on both sides of every pin over the entire cross-section. These undercuts can be used subsequently in the second process step for the production of the connections based on form-fit.

\section{Multimaterial connection by form-fit}

The joint produced in the joining process is shown in Figure 5. For all connections, a form-fit between the adhesion incompatible materials can be detected. In combination with PP, Figure 5a, the pin structures of the PA66 remain stable during the vibration welding process. There is no visible deformation or melting of the pins. In contrast to this, softening and partial melting of the PA66 pins during the joining process occur for combinations with PC, Figure 5c. This dimensional change of the structure is caused by the resulting high joining zone temperatures of around $240^{\circ} \mathrm{C}$, which is near the melting peak temperature of the used PA66. Due to the applied joining pressure, flattening of the pin structure takes place and PA66 melt is partially displaced by the vibration movement into the pin gaps. For joints between PA66PMMA, no melting of the pins can be detected despite joining zone temperatures of around $220^{\circ} \mathrm{C}$. However, a slight flattening of the pin structures could occur during the joining process.

The filling of the structures by the bonding-partner takes place equally for the combinations between
PA66-PP as well as PA66-PMMA. Generally, for both links an almost complete filling of the structures occurs. Exclusively small air inclusions remain, especially in the pin-foot area. In contrast, the multimaterial combination PA66-PC shows a worse filling of the structure. On the one hand, this can be attributed to the high degree of softening and partial melting of the PA66 pins and thus partially closing of the structural depressions, Figure $5 \mathrm{c}$ middle area. On the other hand, this may also be caused by poorer flow behavior of the melt due to thermomechanical properties (e.g. higher viscosity), Figure 5c right-hand side. Despite the thermal influence, a slight form-fit between the partners can also be achieved for this material combination, which enables the formation of the connection based on mechanical adhesion.

\subsection{Melting and softening behavior \\ Heating behavior (DSC)}

Based on results from the DSC analysis, Figure 6 shows the melting and softening behavior of the used thermoplastics. For a heating rate of $10 \mathrm{~K} / \mathrm{min}$, the used PA66 shows a melting peak temperature of $261^{\circ} \mathrm{C}$. When exceeding the peak onset temperature of about $229^{\circ} \mathrm{C}$, melting of the material begins. The melting peak of the investigated $\mathrm{PP}$ is at $162^{\circ} \mathrm{C}$, the onset temperature occurs already at about $105^{\circ} \mathrm{C}$. The assumption of Vetter and Ehrenstein [15] for the maximum joining zone temperature applies to the

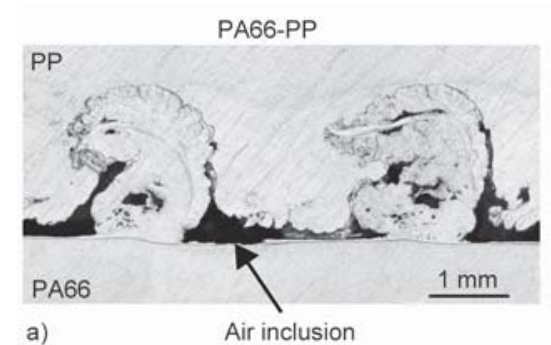

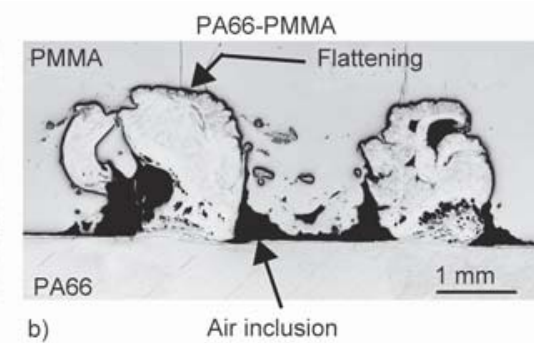

b)

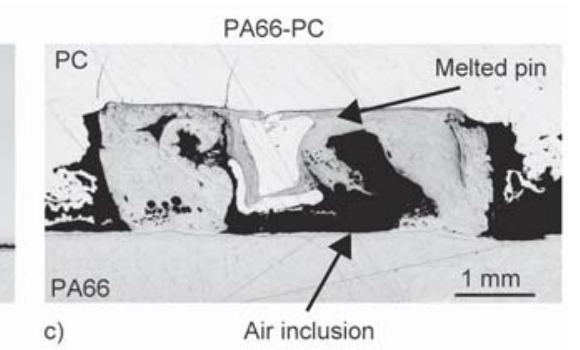

c)

Figure 5. Microscopic image of adhesion incompatible multimaterial bonds based on form-fit between PA66 and PP (a), PA66 and PMMA (b), PA66 and PC (c). 


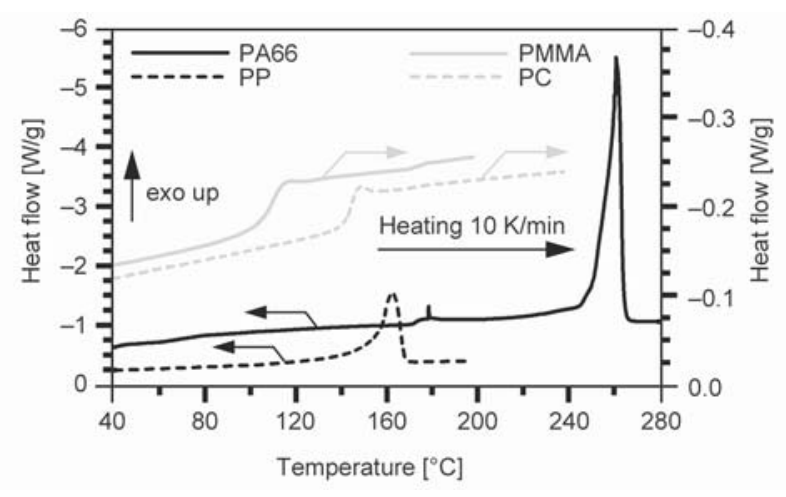

Figure 6. Melting (semi-crystalline) and softening (amorphous) behavior of used materials.

PA66-PP multimaterial combination produced by conventional vibration welding. The constant joining zone temperature is set at $174{ }^{\circ} \mathrm{C}$ and thus lies within the temperature range of $160-190^{\circ} \mathrm{C}$ expected by DSC measurements. Due to this resulting temperature, which is clearly below the melting peak of the structured-partner, the PA66 pin structures stay stable and are not affected during the joining process, as seen in the investigations in Section 3.2. Melting and deformation of the introduced pin structures is expected by exceeding joining zone temperatures about $260^{\circ} \mathrm{C}$. In the range of $230-260^{\circ} \mathrm{C}$, an influence on the geometrical dimension of the pin structures may occur, based on the detected peak onset temperature of the PA66.

The two amorphous thermoplastics PMMA and PC show, contrary to the semi-crystalline materials, exclusively a softening of the material. The softening of the PMMA takes place in the range of 103 to $113^{\circ} \mathrm{C}$, with a glass transition temperature $T_{\mathrm{g}}$ of $108^{\circ} \mathrm{C}$. PC softens at higher temperatures in the range of 143 to $149^{\circ} \mathrm{C}$, with $T_{\mathrm{g}}$ at $146^{\circ} \mathrm{C}$. Inference for the joining zone temperature is not possible for the amorphous materials by using DSC analysis. The resulting temperatures during the conventional vibration welding process, at $228^{\circ} \mathrm{C}(\mathrm{PMMA})$ and $242^{\circ} \mathrm{C}(\mathrm{PC})$, are well above the softening temperature of the polymers. On the one hand, this can be caused by the used heating rate of $10 \mathrm{~K} / \mathrm{min}$, which is significantly below the heating rate of up to $380 \mathrm{~K} / \mathrm{s}$ occurring in the vibration welding process. On the other hand, an introduction of friction energy is also possible above the glass transition and the occurring softening due to the entropy-elastic material behavior. According to Mezger [28], the macromolecules in amorphous thermoplastics become more movable when glass transition is reached. However, the softening process takes place merely gradually with increasing temperature. A fundamental change in the rheological behavior only occurs when the loss modulus of the material exceeds the storage modulus. At this point, the polymer chains slide increasingly on each other and the material exhibits a flowable, predominantly viscous behavior. This material behavior results in a reduction of the internal friction forces of the polymer as reported by Mezger and therefore it can be assumed that a decrease of the introduced friction energy takes place in the vibration welding process at this point.

\section{Heating behavior at process-related heating rates (FSC)}

The results of the FSC analysis show possible influences caused by high process heating rates (Figure 7). Increasing heating rates lead to a shift in the melting or softening temperature to slightly higher temperature regions. The PP melt peak temperature rises slightly from $131^{\circ} \mathrm{C}$ for a heating rate of $100 \mathrm{~K} / \mathrm{s}$ to $133^{\circ} \mathrm{C}$ for an increased heating rate of $500 \mathrm{~K} / \mathrm{s}$. The lower temperature compared to the DSC study can be presumably affiliated to the high cooling rate of $1 \mathrm{~K} / \mathrm{s}$. According to Schawe [29], the melting peak of polypropylene decreases with increasing cooling rates. The two amorphous materials show a shift in their softening behavior towards higher temperatures compared to the DSC. For heating rates of $100 \mathrm{~K} / \mathrm{s}$, the softening of PMMA occurs is in the range of 112 $120^{\circ} \mathrm{C}$ and for $\mathrm{PC}$ between $153-156^{\circ} \mathrm{C}$. An increased heating rate of $500 \mathrm{~K} / \mathrm{s}$ results in a slight increase of

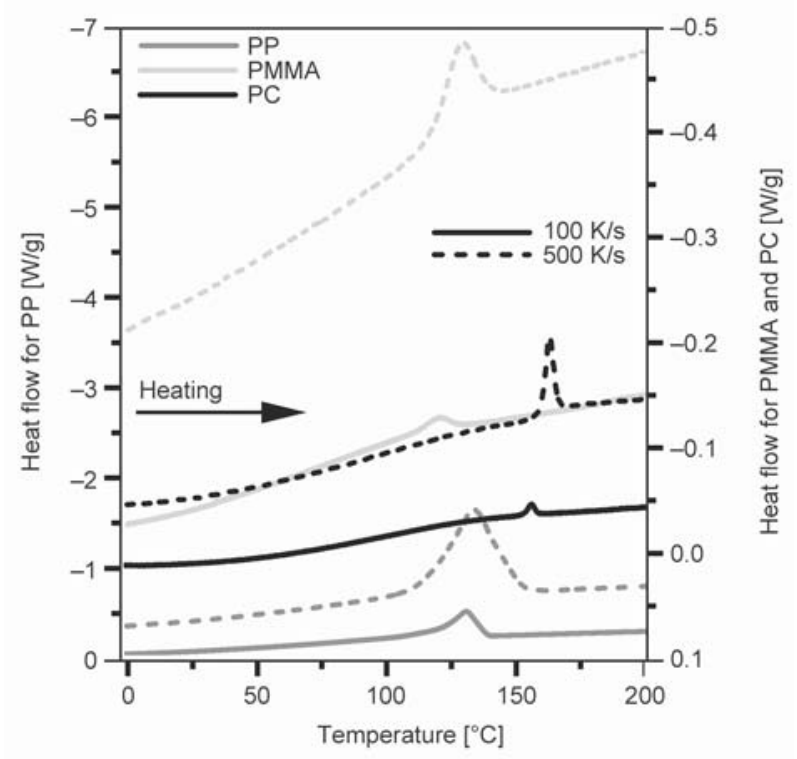

Figure 7. Heating behavior at process-related heating rates after cooling with $1 \mathrm{~K} / \mathrm{s}$. 
the softening temperature range to $114-130^{\circ} \mathrm{C}$ (PMMA) and $157-163^{\circ} \mathrm{C}(\mathrm{PC})$ respectively.

Despite the process-related heating rates, the melting or softening temperatures of the used bonding-partners is plainly below the melting temperate of the polyamide 66 . Nevertheless, the resulting joining zone temperature for combinations with the amorphous thermoplastics are well above the softening temperature and for PC even softening and partial melting of the PA66 takes place. Due to only a small shift in the glass transition for higher heating rates, this is presumably caused by the entropy-elastic material behavior. Owing to this behavior, friction energy can still be introduced into the joining zone even after softening of the amorphous bonding-partner. The polymer chains have a general mobility, once the glass transition temperature has been reached. However, only when the loss modulus exceeds the storage modulus, an increased sliding of the chains on each other takes place [28]. This leads to a clear reduction of internal friction forces only at higher temperatures. For combinations with PC, compared to PMMA, it is assumed that more friction energy is introduced and consequently a higher joining zone temperature results. This may be due to differences in viscosity between the two polymers. According to [15], higher viscosities lead to higher joining zone temperatures in vibration welding.

\subsection{Thermomechanical material properties}

The investigation of the stiffness of the used amorphous thermoplastics as a function of temperature and frequency is shown in Figure 8. With the help of the storage modulus $G^{\prime}$ and the loss modulus $G^{\prime \prime}$, conclusions about the occurring joining zone temperatures, which are clearly above the softening temperatures, shall be drawn.
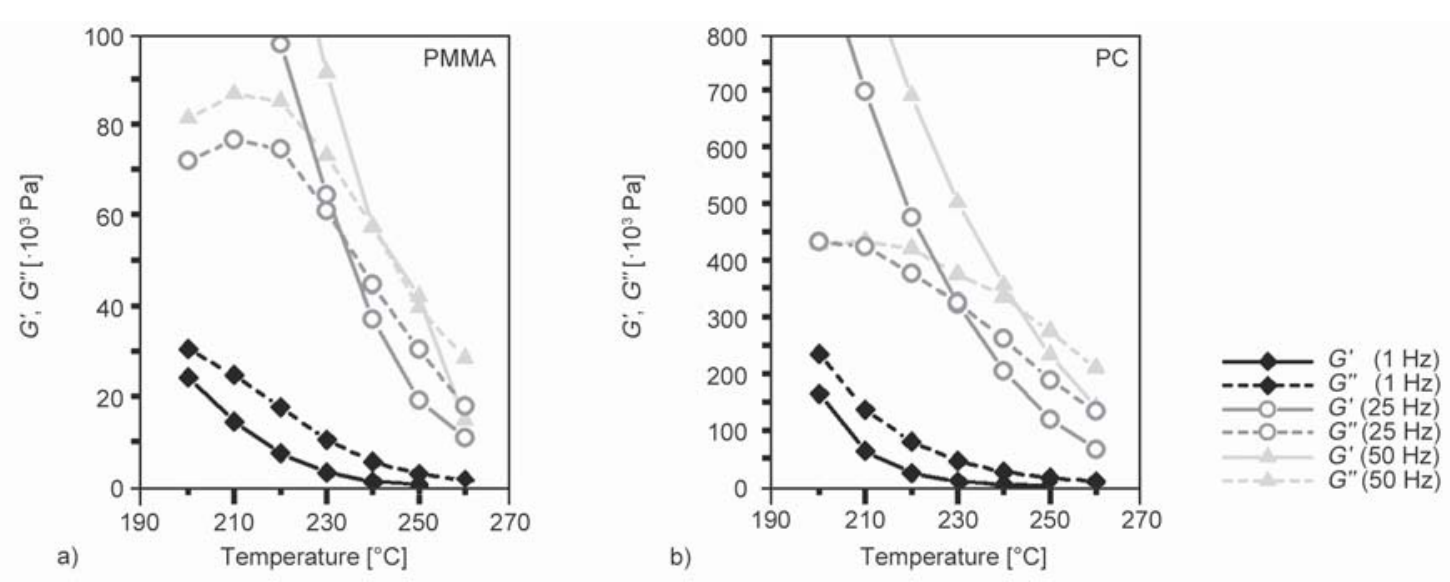

Figure 8. Stiffness of the PMMA (a) and PC (b) bonding-partner depending on temperature and loading frequency.

In general, a shift in the storage and loss modulus to higher values takes place for increasing frequencies. Rising temperatures result in a decrease of both moduli. The intersection point between the storage and loss modulus for PMMA at a load frequency of $1 \mathrm{~Hz}$ is below $200^{\circ} \mathrm{C}$. For higher frequencies, the intercept rises to a temperature slightly above $230^{\circ} \mathrm{C}(25 \mathrm{~Hz})$ and to $240^{\circ} \mathrm{C}(50 \mathrm{~Hz})$. The polycarbonate shows an intersection slightly below $200^{\circ} \mathrm{C}$ at $1 \mathrm{~Hz}$. This intercept increases to about $230^{\circ} \mathrm{C}$ at $25 \mathrm{~Hz}$ and slightly above $240^{\circ} \mathrm{C}$ at $50 \mathrm{~Hz}$. Between the glass transition temperature and the intersection point of storage and loss modulus, amorphous materials show an entropyelastic material behavior [28]. In this area, there is a superposition of elastic and viscous behavior. According to Mezger [28], the material behaves predominantly elastic when the storage modulus is greater than the loss modulus. At the point of intersection between storage and loss modulus the elastic components are equal to the viscous components. As soon as the loss modulus overweighs the storage modulus, the material acts predominantly viscous and the amorphous thermoplastic is flowable. Due to the reduction of the internal friction forces of the polymer with the decrease of the loss modulus, the material exhibits a reduced stiffness with increasing temperature. It can therefore be assumed that above the temperature where storage and loss modulus intersect, the residual stiffness of the melt is greatly reduced due to the predominantly viscous material behavior. For this reason, the energy equilibrium in vibration welding with constant joining zone temperature can be supposed in this area.

Hence, it is assumed that sufficient friction energy can still be introduced in the weld zone above the softening of the material until a sufficient flowability is achieved and internal friction between the chains is 


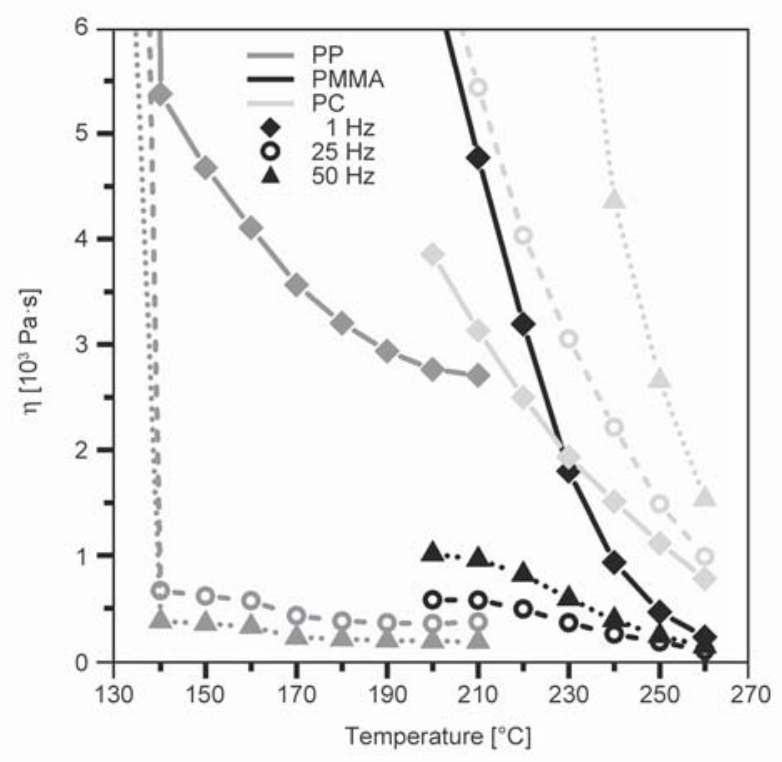

Figure 9. Viscosity of bonding-partner depending on temperature and loading frequency.

reduced. This explains the resulting joining zone temperature well above the glass transition temperature. However, the differences in the resulting joining zone temperature between the two materials PMMA and PC cannot be resolved with the aid of these investigations, because both materials show similar temperatures at their intersection points between storage and loss modulus. Nevertheless, the used PC shows much higher values of the storage and loss modulus compared to PMMA, which may lead to a higher energy input due to the vibration process and therefore to higher weld zone temperatures.

This assumption can also be drawn from the viscosity results shown in Figure 9. In general, a decrease of the viscosity occurs with increasing temperature and frequency for all investigated materials. In the area of the respective joining zone temperature, the materials PP (joining zone temperature $\sim 170^{\circ} \mathrm{C}$ ) and
PMMA (joining zone temperature $\sim 230^{\circ} \mathrm{C}$ ) show similar viscosities of $0.25 \cdot 10^{3} \mathrm{~Pa} \cdot \mathrm{s}(\mathrm{PP})$ and $0.35 \cdot 10^{3} \mathrm{~Pa} \cdot \mathrm{s}$ (PMMA). This might also explain the similar filling of the PA66 structures by these two materials.

PC has a clearly higher viscosity compared to PMMA and PP. As investigations in [15] showed, higher viscosities lead to lower melt volume rates during vibration welding and thus higher temperatures in the welding zone. Due to the higher viscosity of the used PC, a higher resistance against shear exists and so it can be assumed that more frictional energy can be introduced during vibration welding. This leads to higher resulting joining zone temperatures compared to PMMA. Even if the frequency in the vibration welding process is in the range of $230 \mathrm{~Hz}$ and thus higher than the oscillating rheometry measurements, the results still indicate poorer flow properties and poorer filling of the PA66 structures for polycarbonate compared to the other materials used as bondingpartner.

\subsection{Fracture behavior \\ Conventional weld joints}

Due to the adhesion incompatibility of the material combinations, no permanent weld joint could be produced in the conventional welding process. The connections between PA66-PP, PA66-PMMA as well as PA66-PC failed directly after the welding process when removing from the welding system. A bond strength could not be determined.

The fracture surfaces, Figure 10 for the PA66 joining partner, show an adhesive failure for all joints. A mixing and interdiffusion of the joining partners, which occurs in adhesion compatible polymer combinations, cannot be identified. Due to their incompatibility, the joining partners separate directly in the joining zone without material adherences of the respective other

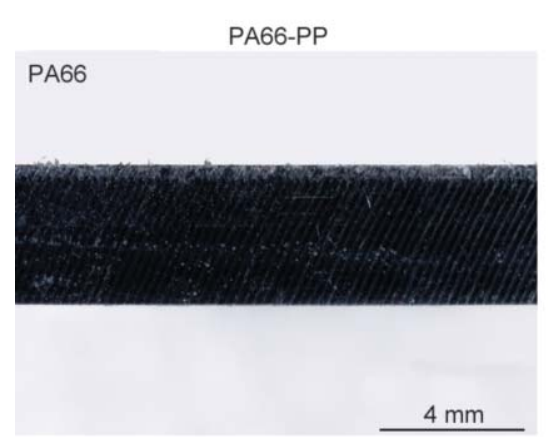

a)

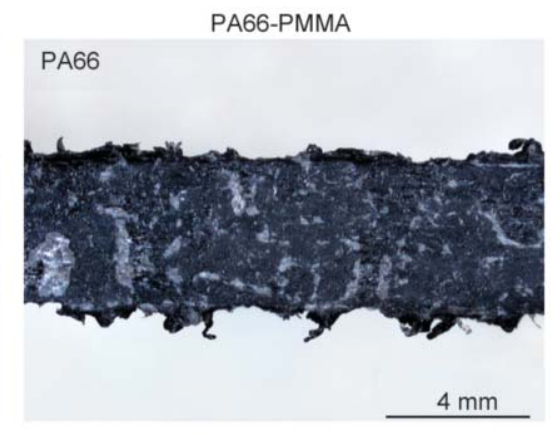

b)

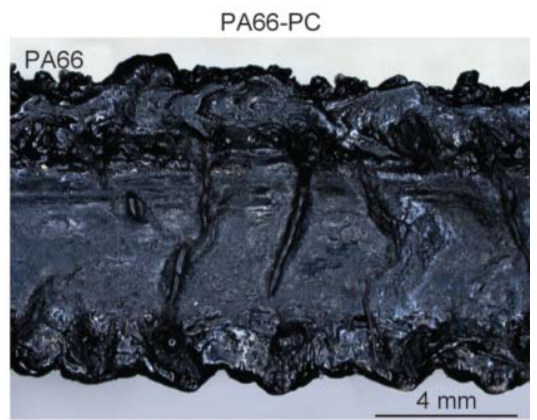

c)

Figure 10. Fracture surfaces of PA66 of bonds produced by conventional vibration welding with PP (a), PMMA (b) and $\mathrm{PC}(\mathrm{c})$ as joining partner. 
partner. Furthermore, a thermal influence of the PA66 (like softening or partly melting of the surface) for combinations with PMMA and PC can be determined, which is caused by the resulting joining zone temperatures. In combination with polycarbonate, melting of the PA66 surface as well as a squeeze flow of the molten polyamide out of the joining zone can be recognized. For combination with polymethylmethacrylate only slight softening on the PA66-surface is visible, a squeeze flow does not occur. Due to the low temperature in the joining zone, no thermal effects on the polyamide occur for links with polypropylene.

\section{Form-fit joints using vibration welding process}

Contrary to the conventional weld joints, the formfit connections exhibit a measurable bond strength, despite their adhesion incompatibility. Figure 11 illustrates the average curves of the different form-fit multimaterial connections during the tensile tests. The PA66-PP link reaches an average tensile force of $433 \pm 39 \mathrm{~N}$ at an elongation of approx. $0.3 \mathrm{~mm}$ until failure occurs. This failure does not take place abruptly; rather a slow decrease of the force at large achievable elongations occurs and a pull-out of the

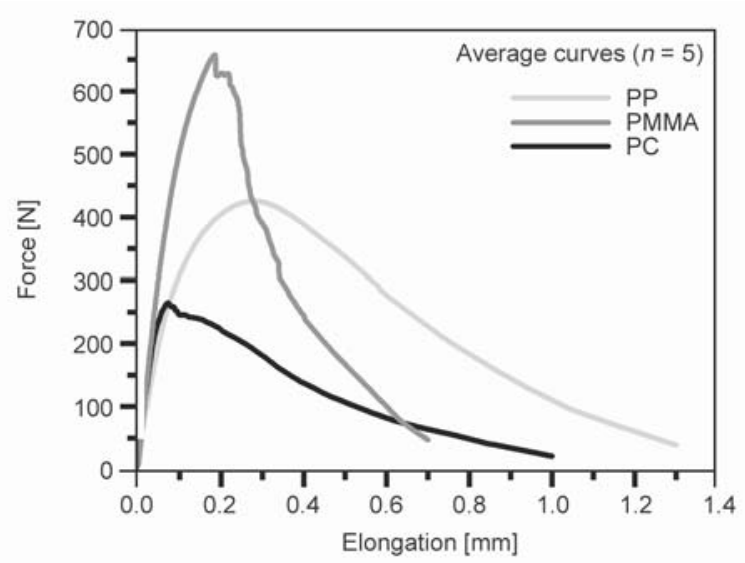

Figure 11. Mechanical behavior of multimaterial connection based on form-fit at tensile load.

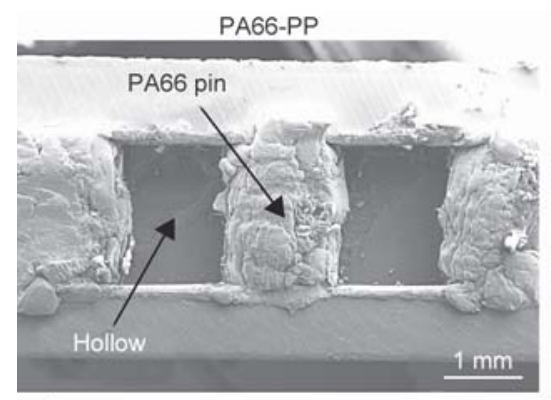

a)

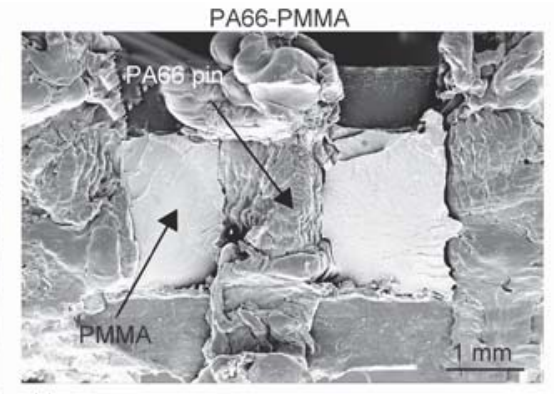

b) polypropylene from the polyamide structures can be determined during the execution of the tensile tests. The achievable strain for completely separation of the joining partners are between $1.0-1.5 \mathrm{~mm}$. In contrast, form-fit bonds between PA66-PMMA show a failure by fracture in the joining zone during the test execution. The average breaking force is $683 \pm 77 \mathrm{~N}$ (at an elongation of approx. $0.2 \mathrm{~mm}$ ) with a resulting elongation for complete separation in the range of 0.5-0.9 mm. Similar to the PA66-PMMA joints, PA66$\mathrm{PC}$ connections also fail by fracture in the joining zone with an average breaking force of $267 \pm 45 \mathrm{~N}$ at elongations between $0.05-0.2 \mathrm{~mm}$. The resulting strain for complete separation of the partners is between $0.7-1.2 \mathrm{~mm}$.

Figure 12 shows the fractured surface of the polyamide by SEM after mechanical testing. For the multimaterial joint between PA66-PP, purely the hollow structures in the polyamide are visible. No residual material is remaining in the PA66 specimen. As the tensile tests suggested, a pull-out of the polypropylene from the polyamide recesses takes place and an adhesive failure occurs. Analogous to the conventional welding tests the PA66 surface does not show a thermal influence on the polyamide during the joining process.

The PA66-PMMA as well as the PA66-PC connection indicate a predominantly cohesive fracture behavior. In combination with PMMA, this failure occurs in the bonding-partner and PMMA material remain in the structural hollows (recesses created by the structuring tool including undercuts) of the PA66. Furthermore, a slight shift of PA66 material to the outside can be recognized. The examination of the structured-partner of the PA66-PC connection reveals the polycarbonate residuals, which remain over the entire joining area of the PA66 specimen. The cohesive failure occurs analogous to the PA66-PMMA connection due to fracture of the bonding-partner.

Figure 12. PA66 surface by SEM after fracture of form-fit connection between PA66 and PP (a), PA66 and PMMA (b), PA66 and PC (c). 
In contrast to the conventional weld joints, the mechanical adhesion of the form-fit joining process enables for all material combination the formation of a bond. The fracture behavior of the investigated multimaterial combinations is thereby mainly affected by the bonding-partner. The abrupt failure of the PA66-PMMA link can be due to the very low possible elongations of the PMMA and the differences between the young's moduli of the materials. This results in a fracture at the smallest surface (beginning of the pin gaps) were stress concentration is likely to be accumulated. In comparison, significant higher possible elongations at break of the material can explain the smaller drop in force at achievable higher elongations for the PA66-PC combination. The fact that the fracture does not occur at the beginning of the pin gaps cannot be justified yet and shall be analyzed in further investigations.

Despite the thermal influence of the pins during the joining process, the combination PA66-PC was able to build a form-fit, which allows the transfer of force during the mechanical characterization. Even with a partial closure of the pin gaps, polycarbonate could still flow into the undercuts, which still were present sufficiently. In case of an increased thermal influence on the structured partner, however, it can be assumed that sufficient form-fit cannot longer be produced (due to a complete closure of the pin gaps and/or the fully destruction of the undercut sections of the pins). This would presumably lead to a different failure behavior or even prevent the generation of a form-fit connection.

\section{Conclusions}

The results obtained within the framework of the present research show that form-fit joints produced in the vibration welding process allow the combination of adhesion incompatible polymer combinations. In particular, the investigations have shown that with the aid of the form-fit, connections between semi-crystalline and amorphous thermoplastics can be produced in welding process. This allows for example the functionalization of technical components with optical polymers, even without using demanding fasteners or adhesives, which require complex pretreatment methods. With the help of the generated butt joint, it could be shown that the form-fit connections can absorb uniaxial loads in direction of the generated pins. Unstructured, conventional vibration welding joints showed no measurable bond strength.
Thus, the undercut is mainly responsible for the existing bonding between the adhesion incompatible multimaterial combinations.

Examinations of the thermomechanical properties showed a predictable temperature in the form-fit joining process for semi crystalline thermoplastics. In contrast, the analysis of the softening behavior of amorphous polymers does not allow any previous estimation of the appearing temperature, which could be attributed to their entropy-elastic behavior above glass transition. Furthermore, the investigations were able to elucidate first dependencies of the resulting bond quality (e.g. deformation of pins, filling of undercuts, fracture behavior) of the form-fit multimaterial connection on the material properties of each partner (bonding-partner and structured-partner) in their combination. In addition to the requirement of creating pin structures with undercuts in the structuredpartner, a sufficient stability of these structures should exist during the joining process. A melting of the structured-partner results in a destruction of the pins and thus in a reduction of the undercut or partial closing of the pin gaps. This might have a negative effect on the bond quality and, if the pin gaps are completely closed or the undercuts are fully destroyed, can even inhibit the generation of the form-fit connection. For this reason, the resulting joining zone temperature should not rise well above the peak onset point, where melting of the structured-partner begins.

Further investigations shall analyze the occurring interactions between material, structuring as well as joining process and resulting joint quality in the joining by form-fit using vibration welding technology. Among other things, the influence of the process parameters on the generation of pin structures as well as on the filling of these structures shall be analyzed. In addition, further investigations shall clarify the occurring thermomechanical influences during the process, even for other material combinations. Particular attention shall be paid to the relationships between the rheological material behavior and the joining zone temperature during vibration welding of the amorphous polymers, which have been established with the help of these investigations. Here, further analyses are necessary to specify the existing correlations and resolve remaining ambiguities. Further, temperature measurements have to be carried out during the form-fit joining process to enable a correlation of the resulting temperature with the conventional vibration welding process. 


\section{Acknowledgements}

This research did not receive any specific grant from funding agencies in the public, commercial or not-for-profit sectors. The authors thank the Covestro AG, Evonik Industries AG, Lanxess Deutschland GmbH and Sabic Europe B. V. for providing the used materials.

\section{References}

[1] Ehrenstein G. W.: Handbuch Kunststoff-Verbindungstechnik (in German). Hanser, Munich (2004).

[2] Patham B., Foss H. P.: Thermoplastic vibration welding: Review of process phenomenology and processingstructure-property interrelationships. Polymer Engineering and Science, 51, 1-22 (2011).

https://doi.org/10.1002/pen.21784

[3] Amanat N., James N. L., McKenzie D. R.: Welding methods for joining thermoplastic polymers for the hermetic enclosure of medical devices. Medical Engineering and Physics, 32, 690-699 (2010).

https://doi.org/10.1016/j.medengphy.2010.04.011

[4] Stokes V. K.: Vibration welding of thermoplastics. Part I: Phenomenology of the welding process. Polymer Engineering and Science, 28, 718-727 (1988).

https://doi.org/10.1002/pen.760281104

[5] Pal K., Panwar V., Friedrich S., Gehde M.: An investigation on vibration welding of amorphous and semicrystalline polymers. Materials and Manufacturing Processes, 31, 372-378 (2016).

https://doi.org/10.1080/10426914.2015.1019111

[6] Rotheiser J.: Joining of plastics - Handbook for designers and engineers. Hanser, Munich (1999).

[7] Baldan A.: Adhesively-bonded joints in metallic alloys, polymers and composite materials: Mechanical and environmental durability performance. Journal of Materials Science, 39, 4729-4797 (2004). https://doi.org/10.1023/B:JMSC.0000035317.87118.ab

[8] Grewell D. A., Benatar A., Park J.: Plastics and composites welding handbook. Hanser, Munich (2003).

[9] Stokes V. K.: Vibration welding of thermoplastics. Part II: Analysis of the welding process. Polymer Engineering and Science, 28, 728-739 (1988).

https://doi.org/10.1002/pen.760281105

[10] Schlarb A. K. H., Ehrenstein G. W.: Parameterwahl beim Vibrationsschweißen von Polypropylen (in German). Plastverarbeiter, 43, 46-51 (1992).

[11] Potente H., Michel P., Ruthmann B.: Analysis of vibration welding. Kunststoffe, 77, 31-34 (1987).

[12] Vetter J., Ehrenstein G. W.: On-line quality determination in vibration welding. Kunststoffe, 88, 986-989 (1998).

[13] Stokes V. K.: Vibration welding of thermoplastics. Part III: Strength of polycarbonate butt welds. Polymer Engineering and Science, 28, 989-997 (1988). https://doi.org/10.1002/pen.760281508
[14] Schlarb A. K. H., Ehrenstein G. W.: The impact strength of butt welded vibration welds related to microstructure and welding history. Polymer Engineering and Science, 29, 1677-1682 (1989). https://doi.org/10.1002/pen.760292309

[15] Vetter J., Ehrenstein G. W.: Biaxial vibration welding of polypropylene. in 'Annual Technical Conference ANTEC 99. New York City’ Vol 1, 1344-1348 (1999).

[16] Bates P. J., Kontopoulou M., Sidiropoulos V., Park G., Zou X.: Real time temperature measurement of nylon 66 butt-joints during vibration welding. in 'Annual Technical Conference - ANTEC. Nashville, US', Vol 1, 1073-1077 (2003).

[17] Hopmann C., Weber M.: New concepts for joining dissimilar thermoplastics using laser radiation. Joining Plastics, 2, 116-125 (2012).

[18] Habenicht G.: Kleben. Springer, Berlin, Heidelberg (2009).

[19] Jahn J., Weeber M., Boehner J., Steinhilper R.: Assessment strategies for composite-metal joining technologies - A review. Procedia CIRP, 50, 689-694 (2016). https://doi.org/10.1016/j.procir.2016.05.034

[20] Rodríguez-Vidal E., Sanz C., Soriano C., Leunda J., Verhaeghe G.: Effect of metal micro-structuring on the mechanical behavior of polymer-metal laser T-joints. Journal of Materials Processing Technology, 229, 668677 (2016). https://doi.org/10.1016/j.jmatprotec.2015.10.026

[21] Fuchs A. N., Wirth F. X., Rinck P., Zaeh M. F.: Lasergenerated macroscopic and microscopic surface structures for the joining of aluminum and thermoplastics using friction press joining. Physics Procedia, 56, 801810 (2014).

https://doi.org/10.1016/j.phpro.2014.08.088

[22] Feistauer E. E., Guimarães R. P. M., Ebel T., dos Santos J. F., Amancio-Filho S. T.: Ultrasonic joining: A novel direct-assembly technique for metal-composite hybrid structures. Materials Letters, 170, 1-4 (2016). https://doi.org/10.1016/j.matlet.2016.01.137

[23] Amend P., Pfindel S., Schmidt M.: Thermal joining of thermoplastic metal hybrids by means of mono- and polychromatic radiation. Physics Procedia, 41, 98-105 (2013).

https://doi.org/10.1016/j.phpro.2013.03.056

[24] Lucchetta G., Marinello F., Bariani P. F.: Aluminum sheet surface roughness correlation with adhesion in polymer metal hybrid overmolding. CIRP Annals, 60, 559-562 (2011). https://doi.org/10.1016/j.cirp.2011.03.073

[25] Wolf M., Kleffel T., Leisen C., Drummer D.: Joining of incompatible polymer combinations by form fit using the vibration welding process. International Journal of Polymer Science, 2017, 6809469/1-6809469/8 (2017). https://doi.org/10.1155/2017/6809469 
[26] Rhoades A. M., Williams J. L., Androsch R.: Crystallization kinetics of polyamide 66 at processing-relevant cooling conditions and high supercooling. Thermochimica Acta, 603, 103-109 (2015). https://doi.org/10.1016/j.tca.2014.10.020

[27] Kennedy P., Zheng R.: Flow analysis of injection molds. Hanser, Munich (2013).
[28] Mezger T.: Das Rheologie Handbuch. Vincentz, Hannover (2016).

[29] Schawe J. E. K.: Analysis of non-isothermal crystallization during cooling and reorganization during heating of isotactic polypropylene by fast scanning DSC. Thermochimica Acta, 603, 85-93 (2015). https://doi.org/10.1016/j.tca.2014.11.006 